\title{
Correction: Mortality Due to Malignant and Non-Malignant Diseases in Korean Professional Emergency Responders
}

\author{
Yeon-Soon Ahn, Kyoung Sook Jeong
}

1. There is an error in reference 7. The correct reference is Taeger D, Sun T, Keil U, Straif K. A stand-alone window application for computing exact person-years, standardized mortality ratios and confidence intervals in epidemiologic studies. Epidemiology 2000;11:607-608.

2. In the Methods section, there is an error in the equation in the section titled "Statistical Analysis." The formula is incorrect. Please view the complete, correct formulas and added text here:

$$
\begin{aligned}
& \mathrm{P}\left(\mathrm{X} \geqq x \mid \mu=\mu_{L}\right)=\sum_{k=x}^{\infty}\left(e^{-\mu_{L}} \mu_{L}^{k}\right) / k ! \leqq \alpha / 2, \\
& \mathrm{P}\left(\mathrm{X} \leqq x \mid \mu=\mu_{U}\right)=\sum_{k=0}^{x}\left(e^{-\mu_{U}} \mu_{U}^{k}\right) / k ! \leqq \alpha / 2
\end{aligned}
$$

Where $\mathrm{X}$ is a Poisson random variable with parameter $\mu$ of the Poisson distribution, $x$ is a observed value of $\mathrm{X}$, and $\mu_{L}$ and $\mu_{U}$ are the values of the lower confidence limit and upper confidence limit of $\mu$ respectively, and the $\alpha$ is 0.05 at $95 \% \mathrm{CI}$.

3. A reference is omitted from the last sentence of the first paragraph under the subheading "Statistical Analysis" in the Methods section. The sentence should read: In theory, confidence interval is expressed as following equation at 95\% CI (Sahai et al., 1993). The reference is: Sahai H, Khurshid A. Confidence intervals for the mean of a Poisson distribution: A review. Biom J. 1993;35:857-867.

\section{Reference}

1. Ahn Y-S, Jeong KS (2015) Mortality Due to Malignant and Non-Malignant Diseases in Korean Professional Emergency Responders. PLoS ONE 10(3): e0120305. doi:10.1371/journal.pone.0120305 PMID: 25756281 Mortality Due to Malignant and Non-Malignant Diseases in Korean Professional Emergency Responders. PLoS ONE 10(9): e0139255. doi:10.1371/journal.pone.0139255

Published: September 25, 2015

Copyright: @ 2015 Ahn, Jeong. This is an open access article distributed under the terms of the Creative Commons Attribution License, which permits unrestricted use, distribution, and reproduction in any medium, provided the original author and source are credited. 